\title{
BMJ Open Quality Improving uptake of cervical cancer screening services for women living with HIV and attending chronic care services in rural Malawi
}

\author{
George C Talama, ${ }^{1}$ Mairead Shaw, ${ }^{2}$ Jordan Maloya, ${ }^{1}$ Tafwirapo Chihana, ${ }^{1}$ \\ Lawrence Nazimera, ${ }^{3}$ Emily B Wroe, ${ }^{1}$ Chiyembekezo Kachimanga (1) ${ }^{1}$
}

To cite: Talama GC, Shaw M, Maloya J, et al. Improving uptake of cervical cancer screening services for women living with HIV and attending chronic care services in rural Malawi. BMJ Open Quality 2020;9:e000892. doi:10.1136/ bmjoq-2019-000892

Received 29 November 2019 Revised 19 May 2020 Accepted 25 August 2020

\section{Check for updates}

(C) Author(s) (or their employer(s)) 2020. Re-use permitted under CC BY-NC. No commercial re-use. See rights and permissions. Published by BMJ.

${ }^{1}$ Partners In Health, Neno, Malawi

${ }^{2}$ University of California San Francisco, San Francisco, California, USA

${ }^{3}$ Ministry of Health, Neno, Malawi

Correspondence to Dr Chiyembekezo Kachimanga; chembekachimanga@yahoo. co.uk

\section{ABSTRACT}

Malawi has the second highest age-standardised incidence rate and the highest mortality rate of cervical cancer in the world. Though the prevalence of HIV is currently $11.7 \%$ for Malawian women of reproductive age, cervical cancer screening rates remain low. To address this issue, we integrated cervical cancer screening into a dual HIV and non-communicable disease clinic at a rural district hospital in Neno, Malawi. The project was implemented between January 2017 and March 2018 using the PlanDo-Study-Act model of quality improvement (QI). At baseline (January to December 2016), only 13 women living with HIV were screened for cervical cancer. One year after implementation of the QI project, $73 \%(n=547)$ of women aged 25 to 49 years living with HIV enrolled in HIV care were screened for cervical cancer, with $85.3 \%$ of these receiving the screening test for the first time. The number of women living with HIV accessing cervical cancer services increased almost 10 times (from four per month to 39 per month, $p<0.001$ ). Key enablers in our $\mathrm{Ql}$ process included: strong mentorship, regular provision of cervical cancer health talks throughout the hospital, nationally accredited cervical cancer prevention training for all providers, consistent community engagement, continuous monitoring and evaluation, and direct provision of resources to strengthen gaps in the public system. This practical experience integrating cervical cancer screening into routine HIV care may provide valuable lessons for scale-up in rural Malawi.

\section{PROBLEM}

Even though cervical cancer screening services are available in most parts of Malawi, facilities offering the services continue to report very low rates of women receiving the services such as the case of Neno District. Neno District is a rural district in southern Malawi with a population of about 165000 in $2016 .{ }^{1}$ The district has one district hospital, one rural community hospital and 12 primary care facilities. All primary care facilities refer complicated patients to Neno District Hospital, a facility with both inpatient and outpatient services, including surgical services.
By 2016, Neno District was the only district in Malawi implementing an Integrated Chronic Care Clinic-a clinic managing both HIV and non-communicable diseases (NCDs) patients (mainly hypertension, diabetes, epilepsy and chronic respiratory diseases) regardless of their HIV status in all 14 facilities throughout the district. ${ }^{2}$ Apart from HIV and/or NCD medications, all patients, including women living with HIV, benefited from routine screening during their clinic visit which included screening for malnutrition using anthropometric measurements, tuberculosis and hypertension at every visit, as well as annual screening for diabetes. The screening, which aimed at early detection and timely management of the selected conditions, was protocol based and has been described elsewhere. ${ }^{3}$ By the end of 2016, over 10200 patients were receiving care at the Integrated Chronic Care Clinic. Of these, approximately 7800 were HIV-positive and 2400 were being managed for an NCD (Partners In Health (PIH) internal data, 2016).

In contrast, cervical cancer screening was offered as part of maternal and child health $(\mathrm{MCH})$ services in only eight of the 14 health facilities in Neno District, a separate service from the Integrated Chronic Care Clinics. Cervical cancer screening was done using visual inspection of cervix with acetic acid (VIA) to women aged 25 to 49 years which was in line with the Ministry of Health $(\mathrm{MoH})$ policy at the time. For VIA-negative women, re-screening was recommended at 1 year, and to women living with HIV or HIV negative, re-screening was recommended at 3 years. ${ }^{4}$ Depending on the size of the VIApositive lesion, women were referred to one of the two hospitals-Neno District Hospital or Lisungwi Community Hospital-for cryotherapy or cervical biopsy. ${ }^{5}$ In general, facilities which offered cervical cancer screening 
services were reporting very low numbers of women screened.

Though women living with HIV are at high risk of developing cervical cancer and the $\mathrm{MoH}$ recommendation that women between 25 and 49 years living with HIV should undergo annual screening, it was not offered at the Integrated Chronic Care Clinic. ${ }^{6}$

In 2016, 749 women aged 25 to 49 years living with HIV were routinely receiving care at the Integrated Chronic Care Clinic based at Neno District Hospital. However, the hospital only screened 13 women living with HIV for cervical cancer during the same year, with the total women screened in 2016 at Neno District Hospital only reaching 352 in number.

Using Neno District Hospital as the initial facility, we hypothesised that we could increase the uptake and the quality of cervical cancer screening for women living with HIV by integrating the Integrated Chronic Care Clinic with cervical cancer clinic. Additionally, we hypothesised that the integration of the care would improve overall number of women screened for cervical cancer as well as increase detection of VIA-positive women and lesions suspicious for cervical cancer. We aimed to achieve the target of screening $80 \%$ of eligible HIV-positive women screened for cervical cancer (571 out of the 749 eligible) and aimed to achieve this target within 12 months of full integration of the two services. The project was implemented by the $\mathrm{MoH}$ in collaboration with a local organisation, PIH.

\section{BACKGROUND}

Malawi has the second highest age-standardised incidence rate and the highest mortality rate of cervical cancer in the world. ${ }^{78}$ In Malawi, cervical cancer is the most common cancer in females, contributing to half of all cancers. ${ }^{9}$ Cervical cancer in Malawi is also typically diagnosed in advanced stages, resulting in very low survival rates. A recent study reported that the 5-year cervical cancer survival estimate in Malawi is as low as $2.9 \%$, lower than most African countries. ${ }^{10}$

Cervical cancer secondary prevention remains the most common and preferred method of prevention and control of cervical cancer, especially in low-income and middle-income countries where large scale vaccination against human papilloma virus is not yet feasible and affordable. ${ }^{11}$ Cervical cancer secondary prevention aims to identify and treat pre-malignant lesions, and one common method is VIA and cryotherapy. This method has been shown to be feasible, safe and effective in resource-limited settings. ${ }^{12-14}$ Additionally, it has been demonstrated in many settings that VIA and cryotherapy lower mortality and incidence of invasive cervical cancer in resource-constrained environments. ${ }^{15-17}$

Since 2004, Malawi has been providing cervical cancer prevention under the Malawi National Cervical Cancer Control Programme. VIA and cryotherapy remain the most common method of cervical cancer prevention and control in Malawi. ${ }^{12} 18$ The national target for VIA set by the Malawi National Cervical Cancer Control Programme in 2004 was $80 \%$ for all eligible women. Coverage has incrementally increased from $14 \%$ in 2012 , to $27.3 \%$ in 2015 and $47 \%$ in 2017. Though progress is being made, the mark still falls far below $80 \% .^{12} 18$ Health system challenges contributing to low uptake of cervical cancer screening include unequal distribution of VIA sites with more facilities in urban than rural areas, poor coordination of services within and between health centres, lack of well-trained and mentored VIA providers, and inadequate equipment and supplies for VIA and cryotherapy. ${ }^{18} 19$

Cervical cancer is associated with HIV and is one of the AIDS-defining cancers. ${ }^{6}$ Malawi has one of the highest HIV rates in the world, with an $11.7 \%$ prevalence among women of reproductive age. ${ }^{20}$ However, Malawi has one of the most successful large-scale HIV programmes, with 90\% of all people with HIV being diagnosed in 2018 and, among people living with HIV, $78 \%$ are regularly receiving care in HIV clinics. ${ }^{20}$ Therefore, targeting cervical cancer screening in women living with HIV is a clear strategy to improve the uptake of cervical cancer screening in Malawi, as these women are not only at risk of cervical cancer but can also be opportunistically screened when they access HIV care services.

\section{MEASUREMENTS}

Data was collected from the MoH cervical cancer register to monitor uptake of screening and aggregated into monthly reports by VIA providers. We used these monthly reports to measure the outcomes of interest. To show the impact of the quality improvement (QI) project, we grouped the data into two categories: before the QI project (January to December 2016) and after implementing the QI project (January 2017 to March 2018). The main outcome was proportion of eligible women living with HIV enrolled in the Integrated Chronic Care Clinic who received cervical cancer screening services in the first year after the initiation of the QI project. Since the project may have spillover effect on other aspects of cervical cancer screening services, for example the total number of women utilising cervical cancer services or an anticipated increased detection of women with VIA-positive result or women with lesions suspicious for cervical cancer, other outcomes routinely reported in the cervical cancer monthly reports were also evaluated. These additional outcomes, measured before and after implementation of the QI project, included:

1. Monthly increase in women, regardless of their HIV status, accessing cervical cancer screening services per month.

2. Monthly increase in eligible women, regardless of HIV status, receiving VIA for the first time per month.

3. Increase in the rate of women who are VIA positive and the rate of women with lesions suspicious for cervical cancer. 
Monthly cervical cancer reports were entered, cleaned and analysed using Microsoft Excel 2013. Frequencies, means and proportions were used to describe the outcomes of interest and estimate the changes from baseline data. The t-test was used to show the statistical significance of the changes in outcomes before and after implementation of the quality-improvement project. $\mathrm{P}<0.05$ showed statistical significance.

Before the QI project, baseline data was collected from January to December 2016. On average, every month, 29 women, of whom only three were living with HIV, were screened for cervical cancer. The VIA positivity rate and rate of women suspicious for cervical cancer were $2.2 \%$ and $3.8 \%$, respectively.

\section{Design}

The QI was implemented between January 2017 and March 2018 at Neno District Hospital. The district MoH leadership team tasked the cervical cancer coordinator, clinicians and nurses with finding feasible interventions to increase rates of cervical cancer screening. It was decided that women living with HIV and already enrolled in HIV care in the Integrated Chronic Care Clinic would be screened for cervical cancer by integrating VIA and cryotherapy with HIV care.

Neno District Hospital was chosen as the initial site for integration of VIA and cryotherapy with HIV care before eventually expanding to all of the remaining facilities. In this hospital, the Integrated Chronic Care Clinic was conducted twice a week. VIA was provided on a daily basis as part of integrated MCH services (including family planning, antenatal care and postnatal care) by two community nurses. Cryotherapy was not provided at the time due to breakdown of the cryotherapy machine.

Successful integration of cervical cancer screening services and HIV care required linking two complex units: HIV care in the Integrated Chronic Care Clinic and cervical cancer prevention in the MCH unit. Geographically, these clinics were about $50 \mathrm{~m}$ apart. Eligible women living with HIV were systematically identified among all women attending the Integrated Chronic Care Clinic; this was achieved by modifying the clinic's screening forms to include eligibility for VIA. Consistent with all $\mathrm{MoH}$ facilities, all services in Integrated Chronic Care Clinic and $\mathrm{MCH}$ unit (including VIA) were offered free of charge.

\section{STRATEGY}

A QI approach, using the Plan-Do-Study-Act (PDSA) model was used to improve rates of cervical cancer screening among women living with HIV in the Integrated Chronic Care Clinic. PDSA is a QI framework for identifying and testing changes that may lead to improvement in a programme. These identified changes, called change ideas, undergo a series of implementation steps, which include planning for the change, implementing the change, analysing the data to see if the change has produced any improvement and finally acting on the change with additional adjustments in an iterative fashion.

Initially, an advanced public health nurse (APHN) mentor was identified to coordinate the activities of the QI team. Additionally, the mentor accompanied the VIA providers during screening to focus on quality of care throughout the cervical cancer screening process. Through support from the district clinical leadership, a QI team was established consisting of: the APHN mentor, the hospital matron, a senior clinician, the cervical cancer coordinator, a PIH nursing coordinator, and a monitoring and evaluation officer.

\section{PDSA cycle 1: January 2017 to February 2017}

To identify the root causes of low uptake of cervical cancer screening among women living with HIV and develop solutions, the QI team met with healthcare workers working in the Integrated Chronic Care Clinic and $\mathrm{MCH}$ unit. The main reasons identified for low utilisation of cervical cancer screening among women living with HIV included: lack of community awareness on cervical cancer screening, lack of space to perform VIA and cryotherapy due to the high volume of patients, limited trained personnel in VIA and cryotherapy, lack of knowledge on cervical cancer screening among support staff, fragmented follow-up systems for women who are VIA positive or suspected to have cervical cancer, and insufficient supplies.

Based on the identified challenges, the following change ideas were suggested and implemented:

1. Due to high numbers of patients reviewed on clinic days (over 100 on some days) and limited space within the Integrated Chronic Care Clinic, it was not possible to provide VIA and cryotherapy at the clinic. Therefore, it was agreed that the best solution to provide cervical cancer screening was to design a new clinical pathway for identification and referral to cervical cancer screening services for women living with HIV and attending the Integrated Chronic Care Clinic. In this approach, the screening form used in the Integrated Chronic Care Clinic for screening was modified to include identification of women eligible for VIA. After this identification, eligible women were fast-tracked to receive HIV care and then immediately referred to the MCH unit for same-day VIA. Any HIVnegative women eligible for VIA and receiving NCD care in the Integrated Chronic Care Clinic also benefited from the changes in the new clinical pathway.

2. The cryotherapy machine was repaired and in anticipation of increased demand, supplies needed for VIA and cryotherapy were provided to the $\mathrm{MCH}$ unit from medical stores in the district. The support staff were mentored on how to prepare for the cervical cancer screening clinic, with emphasis placed on sterilisation techniques and the correct supplies to include when packaging VIA and cryotherapy equipment.

3 . Health talks were given by support staff (clerks at the Integrated Chronic Care Clinic and hospital attend- 
ants at $\mathrm{MCH}$ ) before clients were reviewed by clinicians and nurses. To assist with the health talks, easyto-follow health talk aids were provided in the local language. The support staff were initially given a 2-day training on cervical cancer health education. They practised giving health talks to a group of clients in the presence of the mentor and nurses to provide ongoing mentorship and answer any difficult questions from the clients. The nurses could also provide the health talks whenever the support staff were busy.

4. All eligible women living with HIV were referred to the $\mathrm{MCH}$ unit to receive VIA on the same day as the clinic visit. After the health talk and as part of their HIV-NCD clinic visit screening, all women were asked if they wanted to have the same-day VIA, with this information recorded in the clinic medical records. If women wanted same-day screening, they were fast-tracked through HIV-NCD care and a referral letter was written in their personal record book for same-day referral to VIA. After VIA, the results were stamped in the women's personal record book, and the women were asked to show the results to the Integrated Chronic Care Clinic the same day or during the next visit. Women who opted to forego the same-day screening were encouraged to return for screening before their following clinic appointment, and they were also reminded at the next clinic visit.

5. Prior to this QI project, Neno District had a weekly community screening programme for NCDs, HIV and other conditions as well as a weekly health education programme on the local radio. Cervical cancer messaging was also disseminated to the community using these existing programmes.

6. We procured an MoH cervical cancer screening result stamp which was used to VIA results of women in their health record book.

7. Through community engagement, we came to understand that there were many false notions regarding how cervical cancer screening was performed. For example, women thought that during the procedure, their uterus was removed to examine the cervix. We therefore developed culturally appropriate visual aids used to explain the cervical cancer screening procedure.

8. We used the existing continuous professional development meetings held weekly at the hospital for nurses and clinicians as a platform to disseminate ongoing changes and results of the QI project.

In PDSA cycle 1 , the change ideas identified by the QI team, MCH team and the Integrated Chronic Care Clinic teams were implemented within 2 months. In months 1 and 2 of PDSA cycle 1, preliminary data on changes in uptake of VIA was reviewed. During this time, the uptake of VIA by women living with HIV started increasing. However, it was unclear if the women living with HIV and accessing VIA were coming from the Integrated Chronic Care Clinic because the $\mathrm{MoH}$ cervical cancer register did not capture where the women were referred from.
Additionally, few staff were available to conduct VIA. This necessitated a second cycle of the QI project.

\section{PDSA cycle 2: March 2017 to April 2017}

In PDSA cycle 2, the QI team, in consultation with the MCH and Integrated Chronic Care Clinic teams, addressed challenges with recording the source of referrals as well as the limited staff available for VIA and cryotherapy. First, in April 2017, the QI team agreed to add a column to the cervical cancer screening register where they could write the patient identification number and indicate the referral source. This enabled the team to know which women were referred from the Integrated Chronic Care Clinic.

Second, six nurses (two from the MCH unit and four from the Integrated Chronic Care Clinic) received a 5-day nationally accredited VIA and cryotherapy training course in April 2017. In this training, the participants were trained on identifying pre-malignant changes after speculum examination and washing of cervix with $3 \%$ to $5 \%$ acetic acid for at least $1 \mathrm{~min}$. Additionally, they were taught on using double-freeze cryotherapy (with either nitrogen dioxide or carbon dioxide gas) as a method of treating pre-malignant lesions. The detailed method of VIA and cryotherapy is explained elsewhere. ${ }^{4}$ After the training, a schedule was established to ensure that all newly trained staff rotated through the MCH unit to retain recently obtained skills, and that extra nurses were available during Integrated Chronic Care Clinic days.

The QI team continued to monitor the data on all women accessing cervical cancer screening services monthly. To ensure continued success and sustainability of the project, the APHN mentor focussed on training two local leaders of the QI team on the importance of ongoing monitoring and evaluation, and imparting skills to stakeholders (patients, nurses, clinicians and support staff), as well as formulating collective plans of action for when something is not working well.

Beginning April 2017, the QI ideas introduced in PDSA cycle 1 and 2 were adopted as the standard clinical practices at Neno District Hospital, and cervical cancer screening data was collected and reviewed on a monthly basis. The APHN mentor left in June 2017, and the two local leaders assumed leadership of the QI team and continued working with the teams to monitor data and ensure that staff, equipment and materials were available for the programme.

\section{RESULTS}

One year after the modification of the cervical cancer screening register to indicate whether women receiving screening services were referred from the Integrated Chronic Care Clinic (April 2017 to March 2018), 547 out of $749(73 \%)$ eligible women living with HIV received cervical cancer screening services. Among the 547 women, 464 women living with HIV $(85 \%)$ received VIA for the first time ever (table 1). 
Table 1 Uptake of cervical cancer screening services at Neno District Hospital

\begin{tabular}{|c|c|c|c|}
\hline & Baseline* $^{*}$ & End linet & P value \\
\hline $\begin{array}{l}\text { Women living with HIV enrolled in the Integrated Chronic Care Clinic who } \\
\text { received cervical cancer screening services in the first year }(\%, n) \neq\end{array}$ & - & $73 \%(547 / 749) \ddagger$ & \\
\hline Women, regardless of HIV status, receiving VIA for the first time per month $(n)$ & 28.1 & 78 & 0.001 \\
\hline Rate of women who are VIA positive (\%) & $2.2 \%$ & $3.9 \%$ & 0.23 \\
\hline
\end{tabular}

*Baseline: January to December 2016

†End line: January 2017 to March 2018

†From April 2017 to March 2018. We did not have baseline before April 2017 as the cervical cancer screening register did not indicate if HIVpositive women were coming from Integrated Chronic Care Clinic

VIA, visual inspection of cervix with acetic acid.

Overall, there was an increase in the number of women accessing cervical cancer screening services per month, regardless of HIV status, from 29 to 90 per month (table 1 and figure 1). Nearly half of these women came from the Integrated Chronic Care Clinic $(\mathrm{n}=560,43 \%)$. Furthermore, the number of women per month receiving VIA for the first time ever, regardless of HIV status, increased from 28 to 78 per month.

The rate of women who were VIA positive among all women who received cervical cancer screening increased from $2.2 \%$ to $3.9 \% \quad(p=0.23)$. Within the women living with HIV from the Integrated Chronic Care Clinic, the VIA positivity rate was $4.5 \%$. The rate of women with lesions suspicious for cervical cancer decreased from $3.8 \%$ to $0.9 \%(\mathrm{p}=0.91)$.

After the QI project, a total of 34 women were found to be VIA positive, with 18 (52.9\%) referred from the Integrated Chronic Care Clinic. Of these women, 20 (58.8\%) received same-day cryotherapy. Similarly, among the 10 women who had lesions suspicious for cervical cancer,

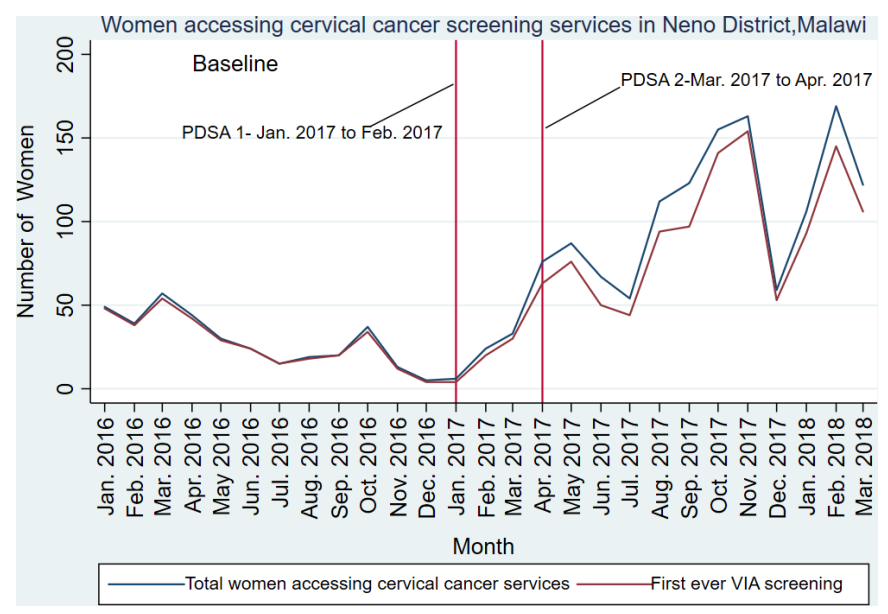

Figure 1 Month-to-month changes in uptake of cervical cancer screening services and first ever cervical cancer prevention using VIA as a screening test in Neno, Malawi. PDSA, Plan-Do-Study-Act; VIA, visual inspection of cervix with acetic acid. five $(50 \%)$ came from women living with HIV from the Integrated Chronic Care Clinic.

The reason for failing to receive same-day cryotherapy included a lack of cryotherapy gas (one woman), a lesion which was too big for cryotherapy (five women) and no cryotherapy provider on the screening day (five women). We could not ascertain the reasons for delaying cryotherapy in the remaining three women. Women with lesions too big for cryotherapy were referred for review and further work-up by the clinician working in the gynaecology clinic at the hospital.

\section{LESSONS AND LIMITATIONS}

The high burden of HIV and cervical cancer, coupled with the low uptake of cervical cancer screening, necessitated the implementation of a QI project to improve the uptake of cervical cancer screening services by women living with HIV in Neno District, Malawi.

Due to high patient volumes, limited space and the existing integration of HIV and NCD services, it was not possible to integrate VIA and cryotherapy within the HIV clinic solely using existing staff. Similarly, many studies have reported that inadequate infrastructure and high patient volumes are two of the main challenges of integrating HIV with cervical cancer screening programmes. ${ }^{21} 22$ Therefore, integration by coordinating HIV services with cervical cancer screening in the MCH unit, through using a coordinated referral and data system, provides another option of integration..$^{23}$ This was what we opted for in this project.

Key enablers of our QI project included: strong mentorship, nationally accredited VIA and cryotherapy training, consistent community engagement and direct provision of resources to strengthen gaps in the public system. These components have also been suggested as solutions in other settings in Malawi. ${ }^{19}$ To address challenges identified during integration of the services, we had to address challenges in the entire system (human resources, supplies and equipment, space, and referral and data systems). However, addressing the majority 
of these challenges did not require extra resources to achieve an impact; a simple re-organisation of existing resources was required. This includes examples such as making small changes to the flow of patients at the Integrated Chronic Care Clinic to identify women living with HIV eligible for VIA, task-shifting to provide routine health talks and actively referring women living with HIV to VIA services, all of which allowed the project to reach $73 \%$ of all eligible women living with HIV in the clinic. This was achieved with minimal investment in local shortterm training, mentorship and materials.

The integration of data systems to measure success was an important lesson. Although the cervical cancer registers record the HIV status of women screened, they do not record the referral source or where women living with HIV obtain HIV care. For effective measurement of integration, we propose adding this data element to the cervical cancer register, as it can help track progress among the enrolled women living with HIV as well as the relative contribution of different referral sources.

The project also encountered some challenges. We found a very low percentage of VIA-positive women compared with other studies, which show high VIA positive rates in HIV-positive women, including $10.4 \%$ in one study in Malawi and 7\% in Tanzania. ${ }^{15}{ }^{24}$ However, another recent study in Malawi found a similarly low VIA positive rate of $3.4 \% .{ }^{16}$ During the evaluation period, we did not investigate why the VIA positivity rate was low. It is possible that the low positivity was due to low cervical cancer rates in this rural cohort of women. However, it is also possible that the quality of the screening was low as VIA is subjective and depends on the skills of the provider. As we continue screening women in this clinic and other clinics in Neno District, we will add additional quality assurance and mentorship in an effort to improve or confirm the low positivity rate. Additionally, not all patients received same-day cryotherapy, and delayed cryotherapy is known to contribute to high loss of follow-up, leaving additional areas for QI work.

The project had some limitations worth mentioning. First, it was implemented in one rural clinic in Neno District Hospital. As a result, the improvements in uptake may not be generalisable to other facilities, especially urban facilities. Future projects can include implementation strategies for more HIV and cervical cancer screening clinics. Additionally, we screened up to $73 \%$ of women within the first year of integration. We did not investigate why we could not reach the rest of the women living with HIV. Finally, we used routinely collected data, with the quality of the data depending on the clinical staff charged with writing programmatic reports.

\section{CONCLUSION}

The project demonstrated the feasibility of improving the uptake of cervical cancer screening services among women living with HIV in rural Malawi using a QI approach, and it provided significant knowledge and experience around integration of cervical cancer screening services into an already complex HIV clinic in rural Malawi with extensive experience in HIV-NCD integration. As far as we know, this is the only project in Malawi to address cervical cancer screening in an HIV clinic in a rural health facility. Two previous studies, which integrated HIV and cervical cancer screening, have been reported in Malawi, both completed in urban and tertiary facilities. ${ }^{16} 17$ This practical experience of integration experience of cervical cancer screening may provide valuable lessons for scale-up in rural Malawi.

Based on these lessons, plans are underway to integrate cervical cancer prevention into Integrated Chronic Care Clinics in the remaining 13 facilities in Neno District. VIA will be done alongside the introduction of thermo-coagulation, an alternative method of treating cervical pre-malignant lesions, which has been shown to be feasible in other settings in Malawi. ${ }^{16}{ }^{24}$ Additionally, lessons from integration with HIV care will be applied to integration with other services, such as for patients with common chronic NCDs, to maximise access to and uptake of cervical cancer screening services in rural Malawi.

Acknowledgements We acknowledge all the clinical officers, nurses and other providers who worked tirelessly to provide care in maternal and child health unit and Integrated Chronic Care Clinic in Neno. We also acknowledge Willy Kwenda and his team who provided cervical cancer training to staff of Neno District.

Contributors GCT, MS, EBW, LN and CK conceptualised the study. GCT, JM and TC collected the data. CK performed data analysis. GCT drafted the first draft. All authors provided feedback and approved the final manuscript for submission.

Funding The authors have not declared a specific grant for this research from any funding agency in the public, commercial or not-for-profit sectors.

Competing interests None declared.

Patient and public involvement Patients and/or the public were not involved in the design, or conduct, or reporting, or dissemination plans of this research.

Patient consent for publication Not required.

Ethics approval The project received ethical clearance from the Malawian National Health Sciences Research Committee, protocol number 1216. Informed consent was not obtained as the review used routinely collected and de-identified data.

Provenance and peer review Not commissioned; externally peer-reviewed.

Data availability statement Data are available upon request.

Open access This is an open access article distributed in accordance with the Creative Commons Attribution Non Commercial (CC BY-NC 4.0) license, which permits others to distribute, remix, adapt, build upon this work non-commercially, and license their derivative works on different terms, provided the original work is properly cited, appropriate credit is given, any changes made indicated, and the use is non-commercial. See: http://creativecommons.org/licenses/by-nc/4.0/.

ORCID iD

Chiyembekezo Kachimanga http://orcid.org/0000-0003-3507-9591

\section{REFERENCES}

1 National Statistical Office. Malawi population and housing census, 2008.

2 Wroe EB, Kalanga N, Mailosi B, et al. Leveraging HIV platforms to work toward comprehensive primary care in rural Malawi: the integrated chronic care clinic. Healthc 2015;3:270-6.

3 Partners In Health. Integrated Care Cascade Toolkit:An Implementation to Guide Screening, Treatment \& Follow-up for HIV and NCDs, 2017.

4 Ministry of Health. Training course in cervical cancer prevention and control in Malawi, 2015. 
5 Kachimanga C, Phiri A, Manase F, et al. Evaluating the use of pathology in improving diagnosis in rural Malawi. Malawi Med $J$ 2018;30:162-6.

6 Shiels MS, Engels EA. Evolving epidemiology of HIV-associated malignancies. Curr Opin HIV AIDS 2017;12:6-11.

7 World Health Organization. Cervical cancer estimated agestandardized incidence rates in 2018, 2018. Available: http://gco.iarc. fr/today

8 Bray F, Ferlay J, Soerjomataram I, et al. Global cancer statistics 2018: GLOBOCAN estimates of incidence and mortality worldwide for 36 cancers in 185 countries. CA Cancer J Clin 2018;68:394-424.

9 Msyamboza KP, Dzamalala C, Mdokwe C, et al. Burden of cancer in Malawi; common types, incidence and trends: national populationbased cancer registry. BMC Res Notes 2012;5:149.

10 Msyamboza KP, Manda G, Tembo B, et al. Cancer survival in Malawi: a retrospective cohort study. Pan Afr Med J 2014;19:234.

11 Bhatla N, Aoki D, Sharma DN, et al. Cancer of the cervix uteri. Int J Gynaecol Obstet 2018;143 Suppl 2:22-36.

12 Msyamboza KP, Phiri T, Sichali W, et al. Cervical cancer screening uptake and challenges in Malawi from 2011 to 2015: retrospective cohort study. BMC Public Health 2016;16:806.

13 Sankaranarayanan R. Screening for cancer in low- and middleincome countries. Ann Glob Health 2014;80:412-7.

14 Shiferaw N, Salvador-Davila G, Kassahun K, et al. The Single-Visit approach as a cervical cancer prevention strategy among women with HIV in Ethiopia: successes and lessons learned. Glob Health Sci Pract 2016;4:87-98.

15 Anderson J, Wysong M, Estep D, et al. Evaluation of cervical cancer screening programs in Côte d'Ivoire, Guyana, and Tanzania: effect of HIV status. PLoS One 2015;10:e0139242.
16 Pfaff C, Singano V, Akello H, et al. Early experiences in integrating cervical cancer screening and treatment into HIV services in Zomba central Hospital, Malawi. Malawi Med J 2018;30:211-4.

17 Phiri S, Feldacker C, Chaweza T, et al. Integrating reproductive health services into HIV care: strategies for successful implementation in a low-resource HIV clinic in Lilongwe, Malawi. J Fam Plann Reprod Health Care 2016;42:17-23.

18 Ministry of Health. 2017 Malawi national cervical cancer control programme annual report, 2018.

19 Maseko FC, Chirwa ML, Muula AS. Health systems challenges in cervical cancer prevention program in Malawi. Glob Health Action 2015;8:26282.

20 UNAIDS. Country factsheets Malawi: 2018 HIV and AIDS estimates, 2018. Available: https://www.unaids.org/en/regionscountries/ countries/malawi

21 Moon TD, Silva-Matos C, Cordoso A, et al. Implementation of cervical cancer screening using visual inspection with acetic acid in rural Mozambique: successes and challenges using HIV care and treatment programme investments in Zambézia Province. J Int AIDS Soc 2012;15:17406

22 Plotkin M, Besana GVR, Yuma S, et al. Integrating HIV testing into cervical cancer screening in Tanzania: an analysis of routine service delivery statistics. BMC Womens Health 2014;14:120.

23 Sigfrid L, Murphy G, Haldane V, et al. Integrating cervical cancer with HIV healthcare services: a systematic review. PLOS One 2017; 12:e0181156.

24 Campbell C, Kafwafwa S, Brown H, et al. Use of thermo-coagulation as an alternative treatment modality in a 'screen-and-treat' programme of cervical screening in rural Malawi. Int $J$ Cancer 2016;139:908-15. 